\title{
Status of direct neutrino mass measurements and the KATRIN project
}

\author{
Kathrin Valerius for the KATRIN collaboration \\ Institut für Kernphysik, Westfälische Wilhelms-Universität Münster, Germany \\ E-mail: valerius@uni-muenster.de
}

\begin{abstract}
In recent years, a number of neutrino oscillation experiments have provided conclusive evidence that neutrinos have non-zero masses. Nevertheless, the absolute scale of these masses which has a fundamental relevance to particle physics and cosmology cannot be deduced from those oscillation experiments. A model independent way to determine the absolute mass scale with a laboratory experiment consists in a precise investigation of the endpoint region of the $\beta$ spectrum (tritium or ${ }^{187} \mathrm{Re}$ ). Recent experiments using tritium have resulted in upper limits for $m\left(\bar{v}_{e}\right)$ of about $2 \mathrm{eV} / \mathrm{c}^{2}$.
\end{abstract}

As a next-generation tritium $\beta$ decay experiment, the Karlsruhe Tritium Neutrino experiment (KATRIN) will increase the sensitivity on $m\left(\bar{v}_{e}\right)$ by one order of magnitude with respect to previous measurements. The experimental set-up includes a windowless gaseous tritium source and two successive electrostatic spectrometers with magnetic adiabatic collimation (MAC-E filter). This talk will give an overview of the direct neutrino mass experiments and report on the current status of the KATRIN project, with major components now being under construction.

International Europhysics Conference on High Energy Physics

July 21 st - 27th 2005

Lisboa, Portugal 


\section{Introduction}

The absolute neutrino mass scale can be determined using different approaches, e.g. via astrophysical observations, searches for neutrinoless double beta decay, or by a precise measurement of the kinematics of weak decays, which is the only model-independent method ${ }^{1}$. In the following, we will discuss two examples of the latter approach, namely

a) investigation of the tritium $\beta$ decay ${ }^{3} \mathrm{H} \rightarrow{ }^{3} \mathrm{He}+\mathrm{e}^{-}+\bar{v}_{e}$ with an electrostatic filter, and

b) bolometric measurements of the decay ${ }^{187} \mathrm{Re} \rightarrow{ }^{187} \mathrm{Os}+\mathrm{e}^{-}+\bar{v}_{e}$ using micro-calorimeters.

\section{Direct electron neutrino mass experiments}

The shape of the electron energy spectrum of nuclear $\beta$ decays contains information on the squared electron antineutrino mass $m^{2}\left(\bar{v}_{e}\right)$. The spectrum is given by

$$
\frac{\mathrm{d}^{2} N}{\mathrm{~d} t \mathrm{~d} E_{e}}=K \cdot F\left(E_{e}, Z\right) \cdot p_{e} \cdot\left(E_{e}+m_{e} c^{2}\right) \cdot\left(E_{0}-E_{e}\right) \cdot \sqrt{\left(E_{0}-E_{e}\right)^{2}-m^{2}\left(\bar{v}_{e}\right) c^{4}},
$$

where $K$ describes the nuclear matrix element, $F$ is the Fermi correction function, $Z$ the charge number of the daughter nucleus, $E_{0}$ the $\beta$ decay endpoint energy, and $p_{e}, m_{e}$ and $E_{e}$ are the momentum, mass and kinetic energy of the electron, respectively. The neutrino mass observable

$$
m^{2}\left(\bar{v}_{e}\right):=\sum_{i}\left|U_{e i}^{2}\right| m^{2}\left(v_{i}\right)
$$

corresponds to an effective squared mass, since experimental sensitivity does not allow to resolve the influence of individual mass eigenvalues $m\left(v_{i}\right)^{2}$. Such kinematic measurements are usually referred to as direct measurements, as they do not rely on further modelling or assumptions. From eq. (2.1) it is evident that the effect of a non-vanishing $m^{2}\left(\bar{v}_{e}\right)$ is largest in the region close to the $\beta$ endpoint energy $E_{0}$, where unfortunately the count rate drops rapidly. To maximise the fraction of relevant count rate, one should use $\beta$ emitting isotopes with a low $Q$-value. ${ }^{187} \mathrm{Re}$ and ${ }^{3} \mathrm{H}$ are particularly suitable since they possess the lowest $\beta$ endpoints known in nature ( $2.5 \mathrm{keV}$ and 18.6 $\mathrm{keV}$, respectively).

\section{$2.1{ }^{3} \mathrm{H} \beta$ decay experiments with electrostatic spectrometers}

Despite the higher endpoint energy as compared to ${ }^{187} \mathrm{Re}$, the use of tritium as a source isotope has several advantages. Since the transition is a super-allowed one, the matrix element becomes independent of the electron energy and the spectral form is entirely determined by phase space. In addition, tritium decays with a short half-life of 12.3 years, compared to the primordial halflife of $4.5 \cdot 10^{10}$ years in the case of the forbidden $\beta$ transition in ${ }^{187}$ Re. Furthermore, the simple electronic shell structure of the tritium atom $(\mathrm{T})$ or molecules involving tritium (e.g. $\mathrm{T}_{2}, \mathrm{THe}^{+}$,

\footnotetext{
${ }^{1}$ Information on neutrino masses can also be inferred from time-of-flight measurements of supernova neutrinos. However, the primary emission time spectra are presently not well understood, and a sub-eV sensitivity cannot be achieved with this method as yet.

${ }^{2}$ Assuming CPT invariance, we do not distinguish between neutrino mass eigenvalues, $m\left(v_{i}\right)$, and the corresponding eigenvalues for antineutrinos, $m\left(\bar{v}_{i}\right)$.
} 
HT) is favourable to the calculation of electronic final states or scattering corrections that need to be applied to eq. (2.1) when using spectrometers.

A comprehensive account of the long history of previous neutrino mass searches using tritium $\beta$ decay by far exceeds the scope of this text ${ }^{3}$. Thus, we will concentrate on two experiments that have been carried out at Mainz, Germany and Troitsk, Russia starting in the early 1990s. Both groups independently developed and installed integrating electron spectrometers of the socalled MAC-E filter type (Magnetic Adiabatic Collimation followed by an Electrostatic filter) [2, 3], a technique which helped to significantly improve the sensitivity on $m^{2}\left(\bar{v}_{e}\right)$ due to an energy resolution and luminosity superior to that of the magnetic spectrometers previously used.

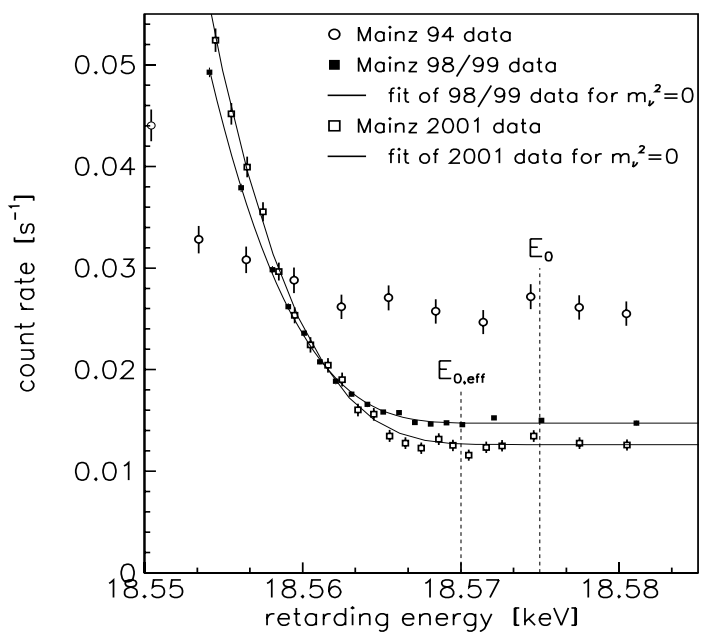

Figure 1: Averaged count rate near the spectral endpoint $E_{0}$ as measured by the Mainz experiment [4]. Data from different measurement periods (1994, 1998/1999, and 2001) are shown for comparison.

The set-up at the Mainz and Troitsk experiments includes similar MAC-E filters $(\Delta E=4.8 \mathrm{eV}$ and $3.5 \mathrm{eV}$, respectively). However, different molecular tritium sources are employed: a thin film of $\mathrm{T}_{2}$ quenchcondensed onto a cold graphite substrate at Mainz, and a windowless gaseous $\mathrm{T}_{2}$ source at Troitsk, giving rise to different kinds of systematic uncertainties. Both experiments took data with comparable signal and background rates after the upgrade of the Mainz set-up in 1995-1997. The endpoint region of the Mainz 1998-1999 and 2001 data is presented in fig. 1 in comparison with the pre-upgrade 1994 data. Inelastic scattering of the electrons within the tritium film, excitation of neighbour molecules caused by the sudden change of nuclear charge in $\beta$ decay, and the self-charging of the tritium film due to the continuous emission of $\beta^{-}$particles represent the main sources of systematic uncertainties of the Mainz experiment. Those have been the subject of detailed investigations which resulted in a good understanding of the systematic corrections. The final refined analysis of the 1998/1999 and 2001 data yields a result compatible with a zero neutrino mass, corresponding to an upper limit on $m\left(\bar{v}_{e}\right)[4]$ :

$$
\begin{aligned}
m^{2}\left(\bar{v}_{e}\right) & =(-0.6 \pm 2.2 \pm 2.1) \mathrm{eV}^{2} / \mathrm{c}^{4} \\
m\left(\bar{v}_{e}\right) & <2.3 \mathrm{eV} / \mathrm{c}^{2}(95 \% \text { C.L. }) .
\end{aligned}
$$

The analysis of the data taken at Troitsk (1994-2001) gives [5]

$$
m^{2}\left(\bar{v}_{e}\right)=(-2.3 \pm 2.5 \pm 2.0) \mathrm{eV}^{2} / \mathrm{c}^{4}
$$

from which an upper limit of

$$
m\left(\bar{v}_{e}\right)<2.05 \mathrm{eV} / \mathrm{c}^{2}(95 \% \text { C.L. })
$$

\footnotetext{
${ }^{3}$ For a recent review see [1], for instance, and references therein.
} 
is deduced. However, the spectra recorded at Troitsk show a small anomaly starting a few eV below the $\beta$ endpoint at a varying position, appearing as a sharp step in the integral count rate. It is assumed to be an experimental artefact that is accounted for in the analysis by adding a single line to the primary $\beta$ spectrum run by run. Additional systematic uncertainties that may be introduced through this procedure are not included in the result quoted in (2.5) and (2.6).

\section{$2.2{ }^{187} \operatorname{Re} \beta$ decay experiments with cryo-bolometers}

Calorimetric searches for a neutrino mass signal in $\beta$ decays provide a strategy complementary to the measurements with electrostatic spectrometers. The basic principle is to use a $\beta$ emitting crystal which itself acts as a particle absorber, where the released $\beta$ decay energy is converted into phonons and, subsequently, can be measured as a small increase in temperature. Working temperatures in the $\mathrm{mK}$ range are needed, which is why cryogenic bolometers are used. In principle, the total $\beta$ decay energy can be measured with this method, except the part carried away by the neutrino. Thus, electronic final state effects are not a matter of concern, and experiments using ${ }^{187} \mathrm{Re}$ with its complicated electronic structure become feasible.

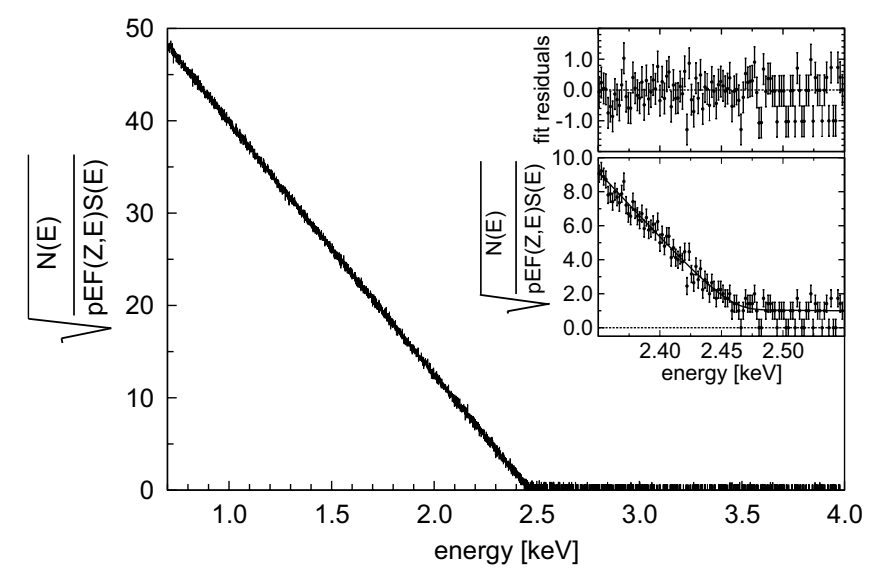

Figure 2: Kurie plot obtained with the MiBeta experiment [7]. $F(Z, E)$ is the Coulomb factor, $S(E)$ the shape factor, while $p$ and $E$ denote the $\beta$ momentum and kinetic energy, respectively.

The aim of such experiments is to exploit the low $\beta$ transition energy in ${ }^{187}$ Re. However, in the bolometric approach, the full range of the $\beta$ spectrum is recorded, while the most sensitive region close to the endpoint is very small. Arrays of several microcalorimeters are implemented to compensate for the intrinsic slowness of thermal detectors (time constants are usually in the range of several hundred $\mu$ s).

Investigations with different forms of rhenium absorbers are presently carried out by the Milan and Genoa groups. The MiBeta experiment (Milan) uses $\mathrm{AgReO}_{4}$ crystals, while the detectors operated in the MANU2 experiment (Genoa) [6] consist of metallic rhenium. MiBeta has reported the lowest neutrino mass limit obtained with cryogenic detectors so far. With an array of 10 micro-calorimeters of an individual energy resolution $\Delta E \approx 30 \mathrm{eV}$ they achieve a $90 \%$ C.L. upper limit of $m\left(\bar{v}_{e}\right)<15 \mathrm{eV} / \mathrm{c}^{2}$ [7]. Figure 2 shows the measured $\beta$ spectrum. To further improve sensitivity, the operation of large arrays of detectors with enhanced energy resolution is required, which is the aim of the MARE project (Microcalorimeter Arrays for a Rhenium Experiment) [8].

\subsection{The KATRIN experiment}

In order to push the sensitivity of neutrino mass experiments using tritium $\beta$ decay towards the sub-eV range, a much larger spectrometer than the ones previously operated is needed, among 
other requirements. The Karlsruhe Tritium Neutrino experiment (KATRIN) [9] with its large main spectrometer ( $10 \mathrm{~m}$ diameter, $23 \mathrm{~m}$ length, $\Delta E=0.93 \mathrm{eV}$ at the tritium endpoint energy) is designed to achieve a sensitivity of $m\left(\bar{v}_{e}\right)<0.2 \mathrm{eV} / \mathrm{c}^{2}(90 \%$ C.L.) after 1000 days of effective measurement time, with statistical and systematic uncertainties contributing about equally. A neutrino mass signal of $m\left(\bar{v}_{e}\right)=0.30 \mathrm{eV} / \mathrm{c}^{2}\left(0.35 \mathrm{eV} / \mathrm{c}^{2}\right)$ will be detected with a significance of $3 \sigma(5 \sigma)$. KATRIN is currently being set up at Forschungszentrum Karlsruhe, Germany, by an international collaboration from the Czech Republic, Germany, Russia, UK and USA. Final commissioning and data taking are scheduled for 2009.

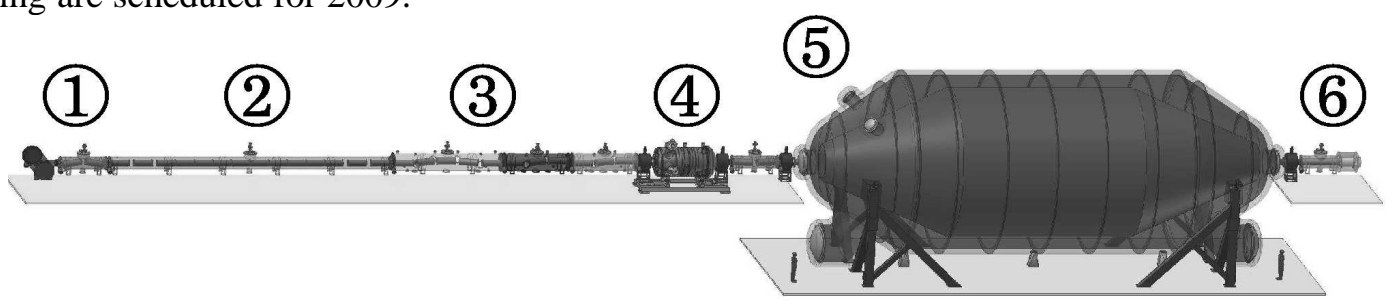

Figure 3: Schematic view of the KATRIN experiment showing the rear monitoring and calibration system (1), the windowless gaseous tritium source (2), the differential and cryopumping electron transport section (3), the pre and main spectrometers $(4,5)$ and the electron detector $(6)$. The total length of the set-up amounts to $\approx 70 \mathrm{~m}$.

The planned experimental set-up is shown in fig. 3. It includes a windowless gaseous tritium source with a daily throughput of $40 \mathrm{~g}$ of molecular tritium, providing an activity of $\approx 10^{11} \beta$ decays per second which yields an enhancement in source strength of about two orders of magnitude with respect to Mainz and Troitsk. Both the amount of $\mathrm{T}_{2}$ and the required high isotopic purity (> 95\%, checked by laser Raman spectroscopy) call for a closed tritium cycle, with the gas being continuously injected to the center of the $10 \mathrm{~m}$ long source tube and differentially pumped at both ends. The source system needs to be kept inside a magnetic field to guide the electrons and at a low temperature with the help of a pressure-stabilised LNe cryostat. The electron transport and cryopumping system consisting of a chain of superconducting solenoids will be placed between the source and the spectrometer section. Its task is to reduce the tritium flow via differential pumping and cryosorption by at least a factor of $10^{11}$, while at the same time guiding the electrons without loss of adiabaticity towards the pre-spectrometer. The cryogenic gas retention concept has been successfully tested with the Tritium Argon Frost Pump experiment (TRAP).

Being essentially a smaller version of the main spectrometer, the pre-spectrometer will act as a coarse energy filter, rejecting all but the last $\approx 200 \mathrm{eV}$ of the tritium $\beta$ spectrum. Thus, only those electrons with energies close to the spectral endpoint (about $10^{3} / \mathrm{s}$ ) will be transmitted towards the entrance of the main MAC-E filter, which already allows a reduction of background. In addition, the spectrometers will be kept at ultra-high vacuum conditions (residual gas pressure $\mathrm{p}<10^{-11} \mathrm{mbar}$ ). The aim is to obtain a low background level of $\lesssim 10 \mathrm{mHz}$ comparable to that at Mainz and Troitsk, however with a much larger spectrometer (inner surface of $\approx 650 \mathrm{~m}^{2}$ ). The actions taken to achieve this include a special choice of construction materials and careful preparation of all surfaces, as well as a bake-out of the whole spectrometer system at a temperature of $350^{\circ} \mathrm{C}$. This general vacuum concept has already been proven at the pre-spectrometer.

Furthermore, a new idea for an active background suppresion which has been successfully tested at the Mainz set-up will be applied to both spectrometers. Background can arise due to 
secondary electrons ejected from the vessel walls (connected to high voltage) by cosmic rays or residual radioactivity. The new concept features an inner electrode made of thin wires which cover the vessel walls. By applying a slightly more negative potential to the wires with respect to the vessel potential, an electrostatic screening is achieved which prevents those secondary electrons from reaching the magnetic flux tube which is mapped onto the detector.

Apart from a high efficiency for electrons at $E_{0}=18.6 \mathrm{keV}$, the detector should also have a low $\gamma$ background. A large array of about 400 PIN photodiodes placed inside a passive shielding and an active veto counter is foreseen.

KATRIN will examine a smaller region of interest below the spectral endpoint as compared to the Mainz and Troitsk experiments. Since two of the main sources of systematic uncertainties, namely the excitation of electronic final states and inelastic scattering processes in the $\mathrm{T}_{2}$ gas, start playing an important role only after a certain energy threshold is passed, their influence can be suppressed by choosing a narrow analysing interval below $E_{0}$. A careful investigation of expected systematic uncertainties has revealed a few contributions with an effect of $\Delta m^{2}\left(\bar{v}_{e}\right) \leq 0.007 \mathrm{eV}^{2} / \mathrm{c}^{4}$ each. One of these takes into account a fluctuation of the retarding high voltage on a $3 \mathrm{ppm}$ level. In order to achieve such a stability, a precision high voltage divider and several calibration sources based on nuclear and atomic standards have been developed and constructed.

\section{Summary}

The search for a neutrino mass signal in the shape of ${ }^{3} \mathrm{H}$ or ${ }^{187} \mathrm{Re} \beta$ spectra provides a modelindependent method complementary to $0 v \beta \beta$ decay experiments. Rhenium $\beta$ decay experiments are in the early stages of development, presently yielding upper limits on the neutrino mass of about $15 \mathrm{eV} / \mathrm{c}^{2}$. With the Mainz and Troitsk tritium $\beta$ decay experiments now having reached their sensitivity limit at about $2 \mathrm{eV} / \mathrm{c}^{2}$, the next step will be to explore the sub-eV region with the KATRIN experiment (designed sensitivity of $0.2 \mathrm{eV} / \mathrm{c}^{2}$ ), which is currently under construction.

\section{Acknowledgments}

The work of the author for the KATRIN experiment is supported by the German Bundesministerium für Bildung und Forschung and within the virtual institute VIDMAN of the Helmholtz Gemeinschaft.

\section{References}

[1] Ch. Weinheimer, C. R. Physique 6 (2005) 768

[2] A. Picard et al., Nucl. Instr. Meth. B 63 (1992) 345

[3] V. M. Lobashev, Nucl. Instr. Meth. A 240 (1985) 305

[4] Ch. Kraus et al., Eur. Phys. J. C 40 (2005) 447

[5] V. M. Lobashev, Nucl. Phys. A 719 (2003) 153c

[6] F. Gatti et al., Nucl. Phys. B 143 (2005) 541

[7] M. Sisti et al., Nucl. Instr. Meth. A $\mathbf{5 2 0}$ (2004) 125

[8] A. Monfardini et al., hep-ex/0509038, and references therein

[9] J. Angrik et al. (KATRIN Design Report 2004), FZK Scientific Report 7090, http://www-ik.fzk.de/katrin 